\title{
Dorsiflexory Wedge Osteotomy to Treat Freiberg's Infraction of the Second Metatarsal Head: A case report
}

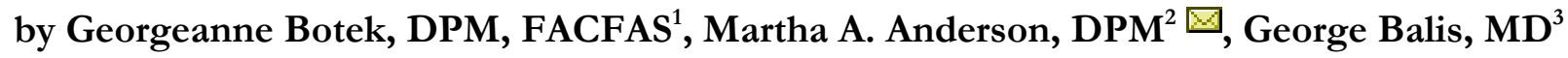

The Foot \& Ankle Journal 1 (11): 3

Freiberg's infraction is an uncommon diagnosis. The incidence of this disorder is unknown. However, it represents the fourth most common intra-articular osteochondrosis. Treatment is based on supportive measures. The underlying pathology is still not well understood. In cases where conservative treatments fail, surgery is indicated to improve patient symptoms. A case of Freiberg's infraction is presented that is treated with joint debridement and dorsiflexory wedge osteotomy of the second metatarsal. This procedure is not technically difficult and affords the patient symptomatic relief by rotating the healthy plantar cartilage of the joint dorsally and decompressing the joint surface.

Key words: Freiberg's infraction, osteochondrosis, osteochondritis dessicans

Accepted: October, 2008

Published: November, 2008

This is an Open Access article distributed under the terms of the Creative Commons Attribution License. It permits unrestricted use, distribution, and reproduction in any medium, provided the original work is properly cited. @The Foot \& Ankle Journal (www.faoj.org)

Freiberg's infraction was first described in $1914{ }^{1}$ This is the only osteochondrosis that is more common in females and has a ratio of $5: 10^{2,3}$ The typical age range at presentation is between 11-17 years. It was originally termed an infraction because trauma was thought to be the cause of insult to the growing and maturing epiphyseal area. Many other inciting events have been proposed and the etiology of this disease now seems to be multi-factorial. ${ }^{4-7}$

Address correspondence to: Martha Anderson, DPM, Kaiser Permanente Department of Podiatry. 12301 Snow Rd. , Parma, OH 44130 .

\footnotetext{
${ }^{1}$ Cleveland Clinic, Medical Director of the Diabetic Foot Clinic, Department of Orthopaedic Surgery, Cleveland, OH. 44130

${ }_{2}$ Third Year Resident (PGYIII), Cleveland Clinic/Kaiser Permanete Residency Program, Cleveland, OH. 44130

${ }^{3}$ Cleveland Clinic, Department of Orthopaedic Surgery, Cleveland, OH. 44130.
}

It is suspected that ischemia to the maturing physis plays a part in disease development. ${ }^{4-7}$ Some authors have also suggested repetitive microtrauma to the affected part may be the causative agent. ${ }^{4-8}$

The second metatarsal head is the most commonly affected and accounts for approximately $68 \%$ of cases. Next is the third metatarsal followed by the fourth and fifth, which are rarely affected. ${ }^{2,6,8-10}$

The most widely used classification system is that of Smillie. ${ }^{13}$ This progresses through the stages of deformity from 1 through 5 based on radiographic findings. 


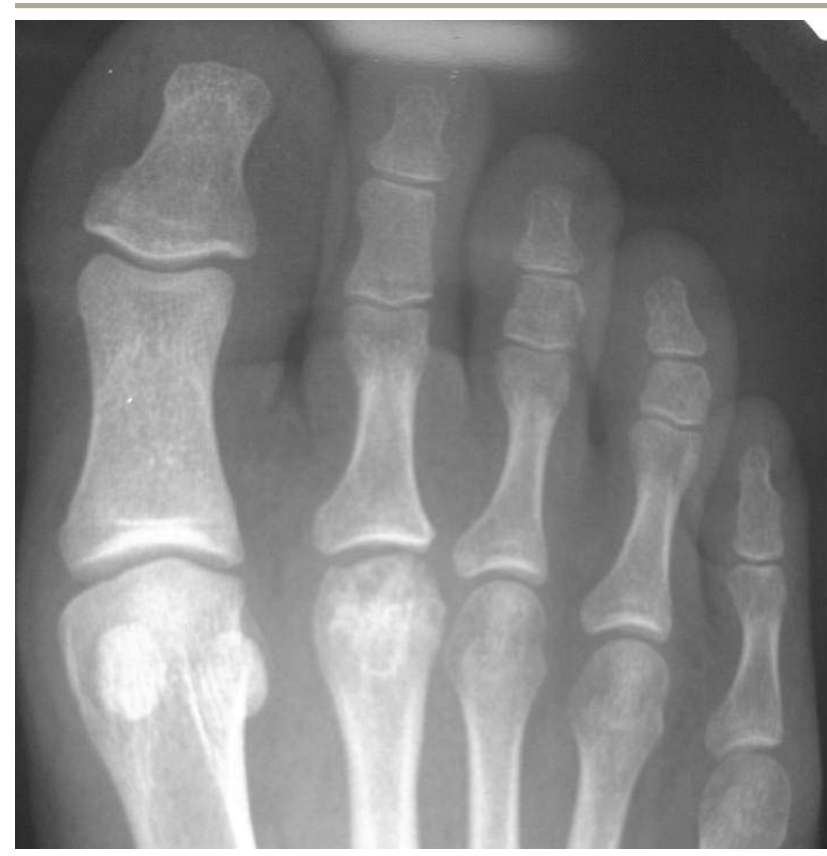

Figure 1 Pre-operative AP radiograph showing Freiberg's infraction with subchondral collapse and joint space narrowing.

Stage 1 of the disease process presents with subchondral epiphyseal fissuring which progresses to collapse of the subchondral plate dorsally with flattening of the articular surface (Stage 3). Late stage disease (4 and 5), there is development of intra-articular loose bodies and degenerative arthrosis with joint space narrowing and metatarsal head flattening. It is also possible to find corresponding degenerative changes at the base of the proximal phalanx in late stage

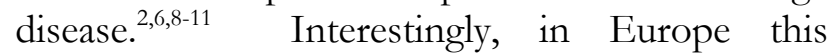
infraction is known as Panner's Disease. ${ }^{4}$

\section{Case Report}

An eighteen year old female college student was seen in our department of orthopaedic surgery for right foot pain of eight months duration. She is diagnosed with Freiberg's infraction of the second metatarsal head. (Figs. 1 and 2)

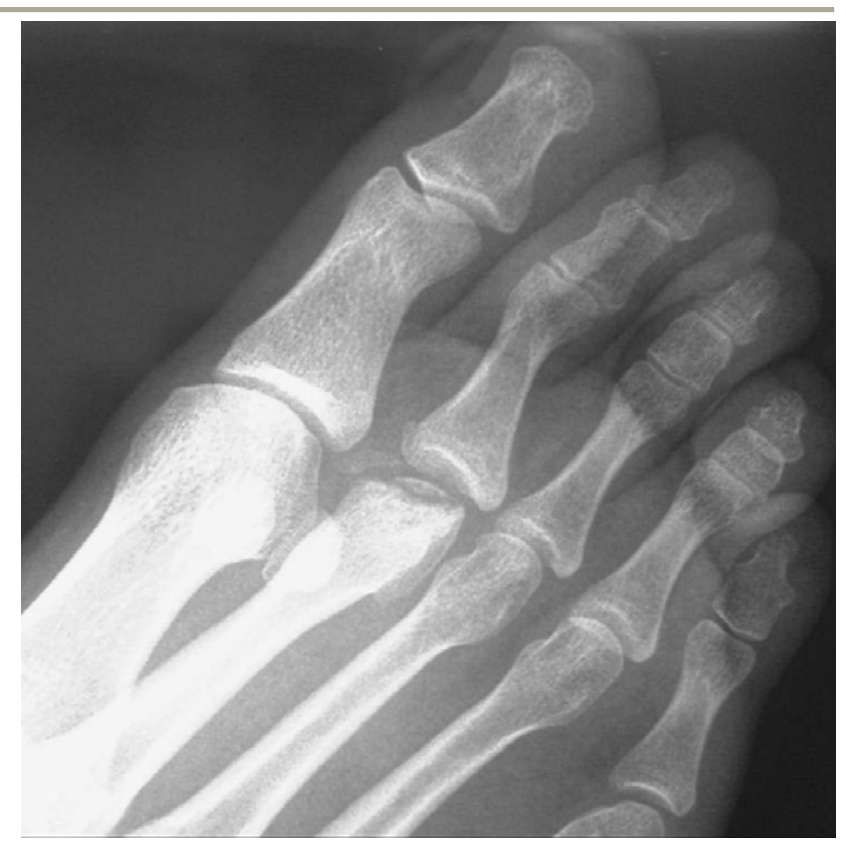

Figure 2 Pre-operative medial oblique showing large intra-articular loose bodies in the second metatarsophalangeal joint.

Pain is localized over the second metatarsophalangeal joint and worsened with weight bearing and activity. Prior to the onset of pain, she was active in the sport of repelling down large rocks and cliffs.

She had previously been seen by a podiatric physician in her home city and various methods of immobilization were tried. She had minimal improvement with a removable CAM walker. No other immobilization or decrease in activity provided pain relief.

At her initial visit to Cleveland Clinic, she was seen by an orthopaedic surgeon who discussed the case with the collaborating author. The patient underwent a corticosteroid injection. A CAM walker was then worn one week after injection. She was then seen in follow-up and reported approximately one month of pain relief after the injection. Further treatments were then considered and a carbon fiber insert was recommended along with a bone stimulator. 


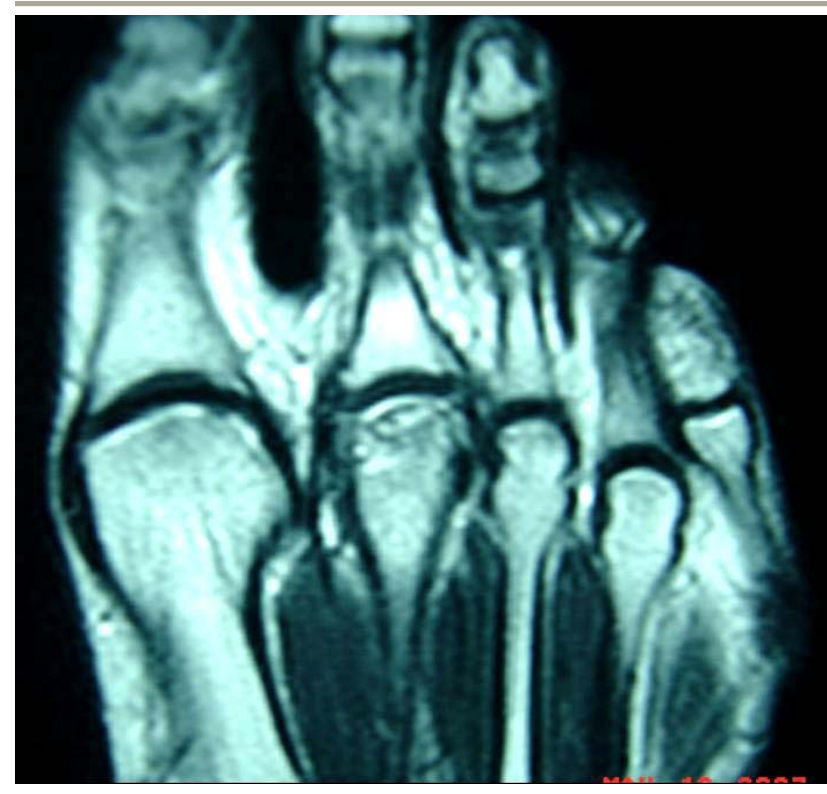

Figure 3 Pre-operative axial T1 weighted MRI showing the adaptive changes and deformity in the metatarsal head.

After using the insert over the course of one month, she developed increasing pain to the affected joint. The bone stimulator did not alter the radiographic appearance of her deformity and did not provide any symptomatic relief.

Serial radiographs were taken throughout the course of treatment which remained unchanged. Magnetic resonance imaging (MRI) was also performed prior to surgery. (Figs. 3 and 4)

\section{Surgical Technique}

A three centimeter incision was placed dorsally over the second metatarsophalangeal joint. Once the capsule was incised and reflected, two large loose bodies were found in the joint measuring approximately three millimeters $(\mathrm{mm})$ each. The dorsal one half to two thirds of the articular cartilage on the metatarsal head was degenerated and atrophic with collapse of the subchondral plate. (Fig. 5)

A dorsiflexory wedge osteotomy of the metatarsal neck was performed to realign the remaining viable plantar articular surface of the metatarsal head.

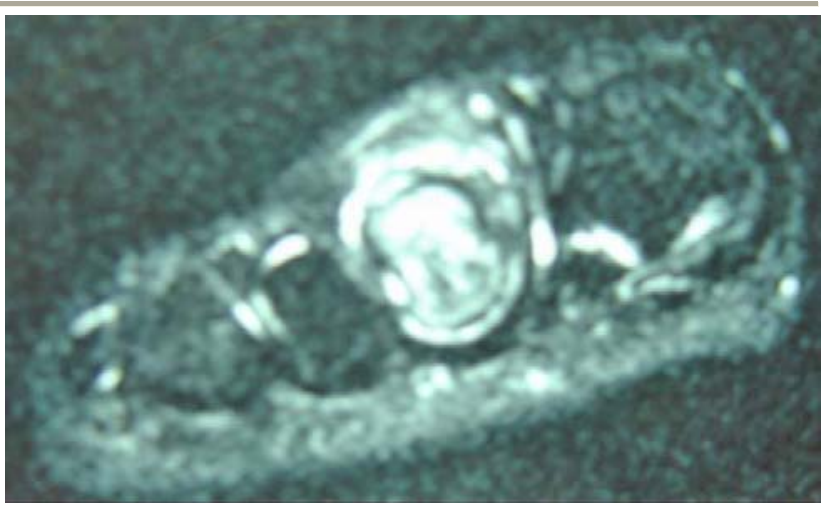

Figure 4 Coronal STIR MRI with edema and inflammatory changes at the second metatarsophalangeal joint.

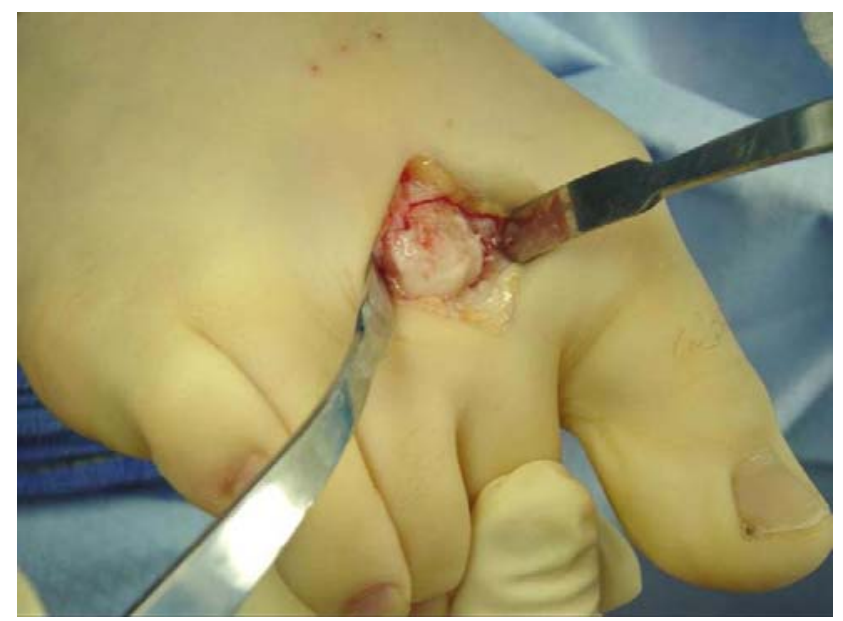

Figure 5 Post debridement photograph showing extensive loss of the dorsal cartilaginous surface of the metatarsal head.

The wedge was taken 1 centimeter proximal to the metatarsal head and was $2-3 \mathrm{~mm}$ in width. Temporary fixation was employed using Kirschner wires. The osteotomy was fixated with a $2.0 \mathrm{~mm}$ cortical screw using standard AO technique.

Proper positioning was determined using intraoperative fluoroscopy after both the temporary and permanent fixation was in place. (Figs. 6A and $\mathrm{B})$. Soft tissues were approximated and a posterior splint with sugar tong was applied.

(c) The Foot \& Ankle Journal, 2008 


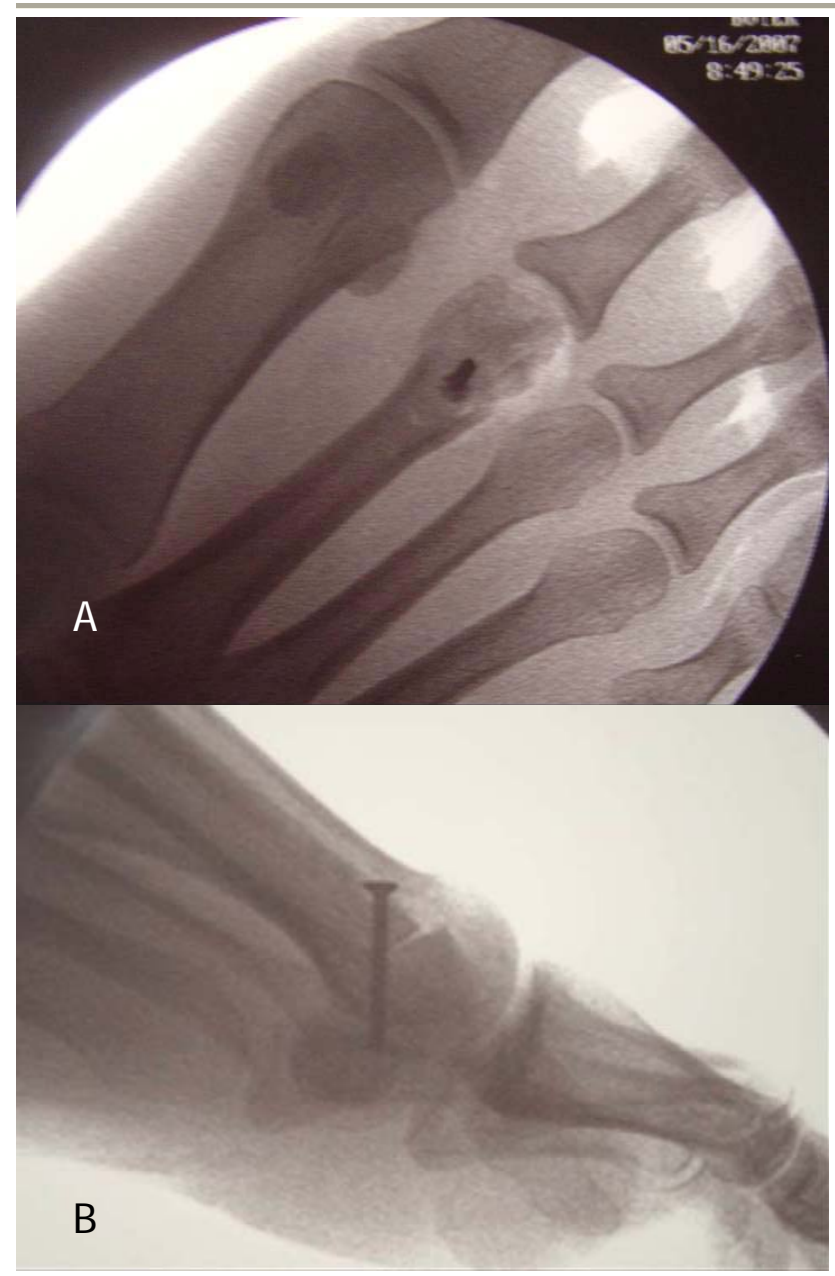

Figures 6A and B Intra-operative fluoroscopy after dorsiflexory closing wedge osteotomy fixated with a $2.0 \mathrm{~mm}$ screw.

The patient was seen in follow up one week postoperatively and placed in a fiberglass, below-theknee cast. At fifteen weeks, post-operative radiographs revealed intact fixation and bony trabeculation across the osteotomy site. (Figs. 7A-C) The patient has minimal discomfort in the second metatarsophalangeal joint and is healing uneventfully. (Fig. 8)

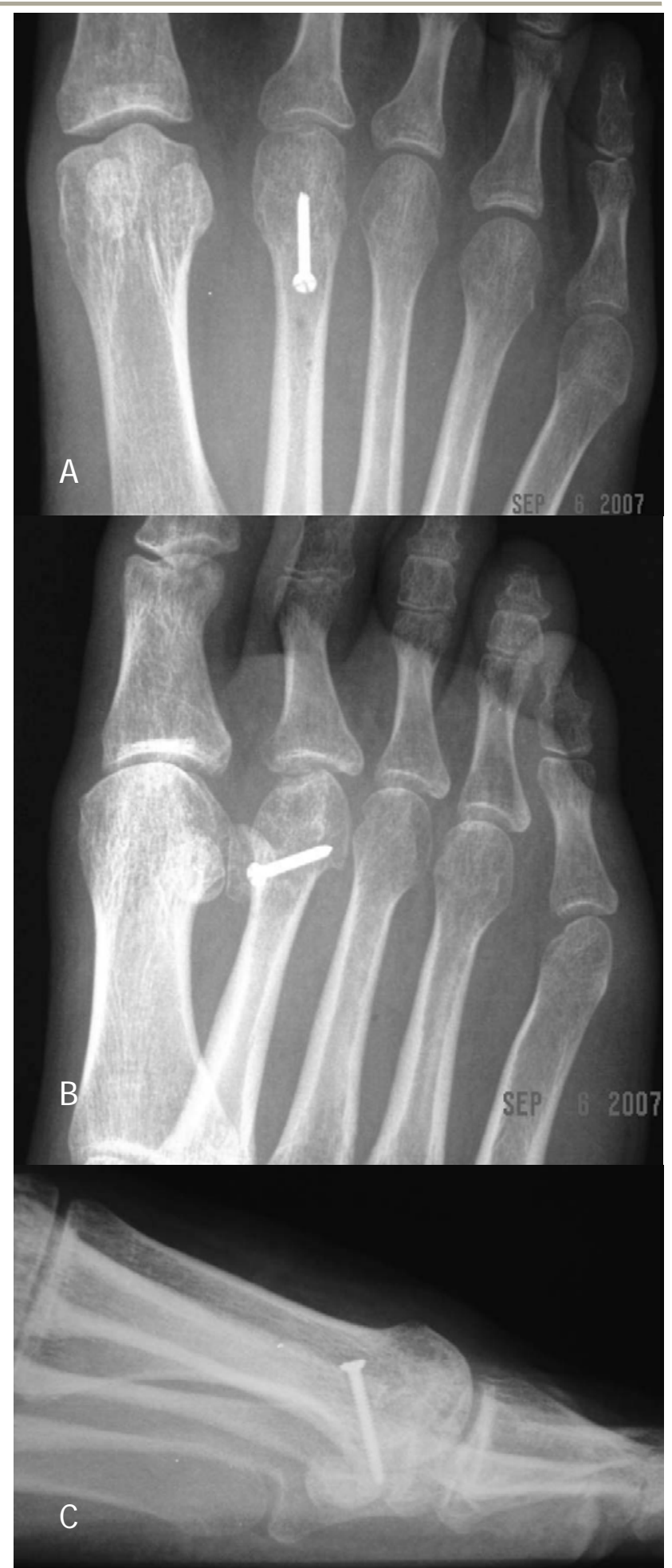

Figures 7 A-C Post-operative radiographs taken fifteen weeks after surgery showing consolidation across the osteotomy site. 


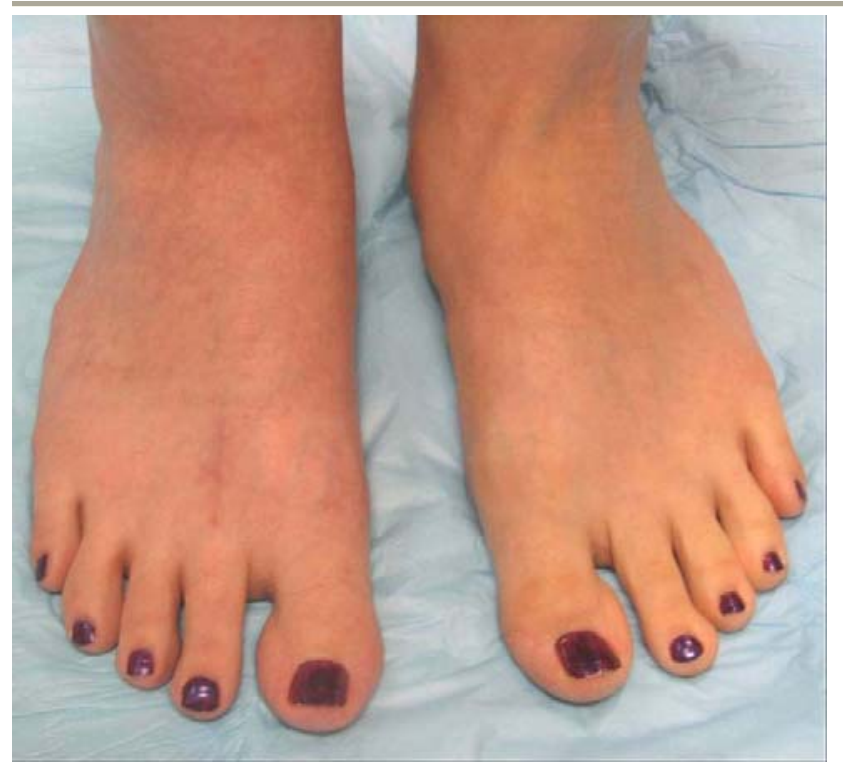

Figure 8 Clinical photograph taken 15 weeks postoperatively showing rectus alignment of the second digit without significant shortening.

\section{Discussion}

Freiberg's infraction is a rare diagnosis and the true incidence is unknown. This is because many patients do not experience symptoms and may have incidental radiographic evidence of disease sequelae. Although it is rare, it is the fourth most common intra-articular osteochondrosis. ${ }^{5,8}$

Many methods of treatment have been described. Immobilization and limitation of activity or shoe modification is the most common course of conservative treatment. ${ }^{2,-6,11}$

Several surgical procedures are also available for patients that fail to improve with conservative means. These include joint debridement, core decompression, metatarsal head resection, joint replacement, bioorthologic grafts and metatarsal osteotomies.

A number of authors have published information on the success of dorsiflexory wedge osteotomies for the treatment of Freiberg's infraction.
This procedure seems to be gaining in popularity due to the successful treatment of all stages in Freiberg's infraction. Gauthier and Elbaz first reported on the technique in $1979 .{ }^{10}$ The osteotomy was intra-articular and excised the devitalized dorsal surface of the metatarsal head. Cerclage wire was employed for fixation. This technique was later modified by Kinnard and Lirette who fixated the osteotomy with absorbable suture. ${ }^{12}$

In 1999, Chao, Lee, and Lin published the extraarticular metaphyseal dorsal closing wedge osteotomy for treatment of Freiberg's infraction. Crossed Kirschner wires were utilized for fixation and were removed in all patients after radiographic signs of healing. Average shortening of the affected metatarsal was $2.1 \mathrm{~mm}$ with this procedure. $^{11}$

The principle behind the dorsiflexory wedge osteotomy is to realign the intact plantar metatarsal cartilage and provide for a more physiologic range of motion. Initially the lesion was excised with an intra-articular osteotomy. This technique is also successful when performing it in an extra-articular fashion. The extra-articular osteotomy has the advantage of being technically less difficult and more stable by the use of compressive screw. It also affords a degree of metatarsal shortening to decompress the involved joint.

\section{Conclusion}

The dorsiflexory wedge osteotomy for the treatment of Freiberg's infraction is a generally successful procedure with good subjective outcomes. It may be performed for all stages of disease. Performing the extra-articular osteotomy allows for ease of fixation compared with the intra-articular osteotomy. This procedure also affords a degree of joint decompression and small amount of metatarsal shortening. 


\section{References}

1. Freiberg AH. Infraction of the second metatarsal bone. Surg Gynecol Obstet (19):163 - 191, 1914.

2. Katcherian DA. Treatment of Freiberg's disease. Orthop Clin N Am 25 (1):69-81, 1994.

3. Duthie RB, Houghton GR. Constitutional aspects of the osteochondroses. Clin Orthop Rel Res (158):19-27, 1981.

4. Ary KR, Turnbo M. Freiberg's infraction an osteochondritis of the metatarsal head. J Am Podiatr Med Assn 69 (2):131-132, 1979.

5. Miller ML, Lenet MD, Sherman, M. Surgical treatment of Freiberg's infraction with the use of total joint replacement arthroplasty. J Foot Surg 23 (1):35-40, 1984.

6. Scartozzi G, Schram A, Janigian J. Freiberg's infraction of the second metatarsal head with formation of multiple loose bodies. J Foot Surg 28 (3): 195-199, 1989.

7. Stanley D, Betts, RP, Rowley DI, Smith TW. Assessment etiologic factors in the development of Freiberg's disease. J Foot Surg 29 (5):444-447, 1990.

8. Binek R, Levinsohn EM, Bersani F, Rubenstein H. Freiberg disease: Complicating unrelated trauma. Orthopedics 11 (5):753-757, 1988.

9. Nguyen VD, Keh RA, Daehler RW. Freiberg's disease in diabetes mellitus. Skeletal Radiol (20) (6):425-428, 1991.

10. Gauthier, $G$ and Elbaz, R. Freiberg's infraction: A subchondral bone fatigue fracture. Clin Orthop Rel Res 142: 93 - 95, 1979.

11. Chao KH, Lee CH, Lin LC. Surgery for symptomatic Freiberg's disease. Acta Orthop Scand 70: 483-486, 1999.

12. Kinnard, P and Lirette, R. Freiberg's disease and dorsiflexion osteotomy. J Bone Joint Surg 73B (5): 864-865, 1991.

13. Smillie IS. Treatment of Freiberg's infraction. Proc R Soc Med. Jan 60(1):29-31, 1967. 